\title{
Diagnósticos de enfermagem frequentes em idosos residentes na área de abrangência de uma Estratégia Saúde da Família
}

\author{
Nursing diagnosis frequently in elderly residents in an area of \\ coverage of family health strategy
}

\section{Diagnósticos de enfermeira frecuentes en los residentes mayores en una area de cobertura de la estrategia salud la familia}

\author{
Recebido: $21 / 09 / 2013$ \\ Aprovado: $31 / 10 / 2013$
}

\author{
Gerson de Souza Santos ${ }^{1}$ \\ Marina Borges Teixeira²
}

O objetivo deste estudo foi identificar os diagnósticos de enfermagem mais frequentes em idosos residentes em uma área de abrangência da Estratégia Saúde da Família, segundo a Taxonomia II da NANDA. Esta é uma pesquisa quantitativa, exploratória e descritiva. Os dados foram coletados durante a consulta de enfermagem por meio de instrumento sistematizado, com 64 idosos, numa unidade de Saúde da Família da cidade de Guarulhos, em 2009. Os idosos eram, em sua maioria, mulheres na faixa etária de 60 a 69 anos, afrodescendentes, sem cônjuge, com baixa escolaridade, aposentadas, com renda de 1 a 3 salários mínimos. Nos 12 domínios existentes houve alterações, por sua vez, os diagnósticos de enfermagem prevalentes foram: estilo de vida sedentário, 53 (82,8\%); interação social prejudicada, 50 (78,1\%); e dentição prejudicada, 46 (71,8\%). 0 Processo de Enfermagem deve ser compreendido como o método para a prática profissional dos enfermeiros que atuam na Estratégia Saúde da Família, possibilitando intervenções mais adequadas aos idosos.

Descritores: Diagnóstico de enfermagem; Idoso; Programa Saúde da Família.

The aim of study was to identify the most frequent nursing diagnoses in elderly residents in an area covered by the Family Health Strategy, according to the NANDA Taxonomy II. This is a quantitative research, exploratory and descriptive. Data collected during the consultation nursing through systematic instrument with 64 elderly, in the health unit of family in the Guarulhos city, São Paulo, Brazil. Both groups were mostly women, aged 60-69 years old, African descendants, no spouse, with low education, retired, earning 1-3 minimum wages. In the 12 dominions were alterations, and the prevalent nursing diagnoses were: sedentary lifestyle, 53 (82.8\%); followed by impaired social interaction 50 (78.1\%); impaired dentition and 46 (71.8\%). The Nursing Process should be understood as the methodological process for the performance of professional practice of nurses working in the Family Health Strategy, enabling the most appropriate interventions for the elderly.

Descriptors: Nursing diagnosis; Aged; Family Health Program.

El objetivo de este estudio fue identificar los diagnósticos de enfermería más frecuentes en los ancianos residentes en un área cubierta por la Estrategia de Salud de la Familia, según la taxonomía NANDA II. Esta es una investigación cuantitativa, exploratoria y descriptiva. Los datos fueron recogidos durante la consulta de enfermería a través del instrumento sistemático, en una unidad de salud de la familia de la ciudad de Guarulhos, São Paulo, Brasil, en 2009. Los ancianos eran en su mayoría mujeres, de 60-69 años de edad, afrodescendentes, sin cónyuge, con bajo nivel educacional, jubilados, ganando de 1-3 salario mínimos. En todos los dominios se observó alteraciones, pero, los diagnósticos de enfermería prevalentes fueron: sedentarismo, 53 (82,8\%); interacción social limitada 50 (78,1\%); y deterioro de la dentadura en 46 (71,8\%). El proceso de enfermería debe ser entendido como método para la realización de la práctica profesional de las enfermeras que trabajan en la Estrategia Salud de la Familia, y que posibilitan mejores intervenciones para los ancianos.

Descriptores: Diagnóstico de enfermería; Ancianos; Programa de Salud Familiar.

1. Enfermeiro. Mestre em Enfermagem. Doutorando em Enfermagem na Universidade Federal de São Paulo. gersonenf@hotmail.com

2. Professora da Universidade de Guarulhos. Doutora em Psicologia Escolar e Desenvolvimento Humano. 


\section{INTRODUÇÃo}

$\mathrm{E}$ m vários países, as populações estão envelhecendo. Estudos mostram que o número de pessoas idosas cresce em ritmo maior do que o número de pessoas que nascem, acarretando um conjunto de situações que modificam a estrutura de gastos dos países em uma série de áreas importantes.

No Brasil, o ritmo de crescimento da população idosa tem sido sistemático e consistente. No período de 1999 a 2009, o peso relativo dos idosos ( 60 anos ou mais de idade) no conjunto da população passou de $9,1 \%$ para $11,3 \%$. Com uma taxa de fecundidade abaixo do nível de reposição populacional, combinada ainda com outros fatores, tais como os avanços da tecnologia, especialmente na área de saúde, atualmente o grupo de idosos ocupa um espaço significativo na sociedade brasileira ${ }^{1}$.

Nesse aspecto, o rápido crescimento da população de idosos no Brasil causa um importante impacto em toda a sociedade, principalmente nos sistemas de saúde. Entretanto, a infraestrutura necessária para responder às demandas desse grupo etário em termos de instalações, programas específicos e mesmo recursos humanos adequados, quantitativa e qualitativamente, ainda é precária ${ }^{2}$.

A Política Nacional de Atenção Básica, regulamentada pela Portaria GM n.o 648, de 28 de março de 2006, se caracteriza por desenvolver um conjunto de ações de saúde, no âmbito individual e coletivo, que abrange a promoção e a proteção da saúde, a prevenção de agravos, o diagnóstico, o tratamento, a reabilitação e a manutenção da saúde. É desenvolvida por meio do exercício de práticas gerenciais e sanitárias democráticas e participativas, sob a forma de trabalho em equipe, dirigidas às populações de territórios bem delimitados, pelas quais assume a responsabilidade sanitária, considerando a dinamicidade existente no território em que vivem essas populações. Utiliza tecnologias de elevada complexidade (conhecimento) e baixa densidade (equipamentos), que devem resolver os problemas de saúde de maior frequência e relevância em seu território. É o contato preferencial dos usuários com os sistemas de saúde. A Estratégia de Saúde da Família visa à reorganização da Atenção Básica no país, de acordo com os preceitos do Sistema Único de Saúde 3 .

Dentro dessa nova ótica de atenção à saúde, o enfermeiro vem desenvolvendo intervenções que buscam maiores e melhores resultados. Com a Estratégia de Saúde da Família, o Sistema Único de Saúde (SUS) passou a incorporar atividades de cunho mais coletivo e abrangente, com perspectivas de, juntamente com outros setores, gerar um impacto significativo na qualidade de saúde e de vida da população idosa 4 .

A assistência sistematizada de enfermagem permite identificar os problemas dos idosos de maneira individualizada, planejar, executar e avaliar o atendimento a cada situação. Para tanto, direcionando a assistência para o nível ambulatorial, a consulta de enfermagem é uma atividade que atende às questões aqui colocadas, e por meio da qual a enfermeira assume a responsabilidade quanto à ação de enfermagem a ser determinada frente aos problemas detectados e estabelece a sua intervenção a partir do diagnóstico ${ }^{5}$.

Para a Associação Norte-Americana dos Diagnósticos de Enfermagem (NANDA), o diagnóstico de enfermagem é um julgamento clínico sobre as respostas do indivíduo, da família ou da comunidade aos problemas de saúde/processos vitais, reais ou potenciais. 0 diagnóstico de enfermagem proporciona seleção das intervenções de enfermagem visando alcançar resultados pelos quais a enfermeira é responsável ${ }^{6}$.

Considerando que o envelhecimento populacional constitui um dos maiores desafios para a Saúde Pública contemporânea, especialmente no Brasil, onde esse fenômeno vem ocorrendo de forma acelerada, o presente estudo teve por objetivo identificar os diagnósticos de enfermagem mais frequentes em idosos residentes em uma área de abrangência de uma Estratégia Saúde da Família, segundo a 
Taxonomia II da NANDA (North American Nursing Diagnosis Association).

\section{MÉTODO}

Trata-se de uma pesquisa descritiva de abordagem quantitativa, realizada em idosos cadastrados numa unidade da Estratégia de Saúde da Família no município de Guarulhos, estado de São Paulo, no período de maio a julho de 2009.

A amostra foi composta por 64 idosos com idade igual ou superior a 60 anos. Os critérios de inclusão na pesquisa foram: aceitar participar da pesquisa por meio da assinatura do Termo de Consentimento Livre e Esclarecido (TCLE), ser idoso (60 anos ou mais), estar cadastrado na Estratégia Saúde da Família - indivíduos de ambos os sexos. Foi determinado como critério de exclusão indivíduos que, após a aceitação da pesquisa e agendamento do dia e horário da coleta de dados, não fossem encontrados no domicílio até a terceira visita consecutiva ou que, em qualquer momento da pesquisa, desistissem de participar desta. Após serem esclarecidos sobre a pesquisa, os idosos concordaram em participar do estudo e assinaram o TCLE. Esta pesquisa foi apreciada pelo Comitê de Ética em Pesquisa conforme Protocolo n. ${ }^{\circ}$ 64/2009.

A coleta de dados foi realizada em um ambiente privativo no domicílio de cada idoso, mediante o preenchimento de um instrumento de coleta de dados elaborado pelos pesquisadores e fundamentado na literatura científica. Este continha aspectos relacionados ao perfil sociodemográfico, de saúde e doença, hábitos alimentares, escala de dor. Foi realizado exame físico clínico, sendo esse o objetivo deste estudo, e os pesquisadores assistiram cada participante conforme as necessidades e diagnósticos de enfermagem identificados. Os dados obtidos foram submetidos à criteriosa análise, sendo identificados os diagnósticos de enfermagem com seus respectivos fatores relacionados e características definidoras. Foi realizada frequência simples dos dados a fim de agrupar os diagnósticos mais frequentes.

\section{RESULTADOS}

Na Tabela 1, pode ser vista a distribuição das características socioeconômicas e demográficas dos pesquisados.

Tabela 1. Características sociodemográficas dos idosos cadastrados numa Estratégia Saúde da Família. Guarulhos, 2009.

\begin{tabular}{|c|c|c|c|c|c|c|}
\hline & \multicolumn{2}{|c|}{ Feminino } & \multicolumn{2}{|c|}{ Masculino } & \multicolumn{2}{|c|}{ Total } \\
\hline & № & $(\%)$ & № & $(\%)$ & $\mathrm{N}$ & $(\%)$ \\
\hline Idade & & & & & & \\
\hline $60-64$ & 06 & 16,2 & 05 & 18,5 & 11 & 17,2 \\
\hline $65-69$ & 09 & 24,3 & 09 & 33,3 & 18 & 28,2 \\
\hline $70-74$ & 07 & 18,9 & 08 & 29,6 & 15 & 23,4 \\
\hline $75-79$ & 05 & 13,5 & 03 & 11,2 & 08 & 12,5 \\
\hline 80 a mais & 10 & 27,1 & 02 & 7,4 & 12 & 18,7 \\
\hline Total & 37 & 100,0 & 27 & 100,0 & 64 & 100,0 \\
\hline Raça referida & & & & & & \\
\hline Branca & 13 & 35,2 & 15 & 55,5 & 28 & 44,0 \\
\hline Negra & 11 & 29,7 & 05 & 18,5 & 16 & 25,0 \\
\hline Parda & 13 & 35,1 & 07 & 26,0 & 20 & 31,0 \\
\hline Total & 37 & 100,0 & 27 & 100,0 & 64 & 100,0 \\
\hline Estado civil & & & & & & \\
\hline Casado & 15 & 40,5 & 14 & 51,8 & 29 & 45,3 \\
\hline Solteiro & 02 & 5,4 & 07 & 26,0 & 09 & 14,0 \\
\hline Viúvo & 18 & 48,7 & 05 & 18,5 & 23 & 36,0 \\
\hline Separado & 02 & 5,4 & 01 & 3,7 & 03 & 4,7 \\
\hline Total & 37 & 100,0 & 27 & 100,0 & 64 & 100,0 \\
\hline Escolaridade & & & & & & \\
\hline Alfabetizado & 12 & 32,4 & 15 & 55,5 & 27 & 42,1 \\
\hline Analfabeto & 25 & 67,6 & 12 & 4,5 & 37 & 57,9 \\
\hline Total & 37 & 100,0 & 27 & 100,0 & 64 & 100,0 \\
\hline
\end{tabular}




Ocupação atual
Aposentado
Sem ocupação
Diarista
Dona de casa
Pintor

Total

1 a 3 salários
4 a 6 salários
Total

Observou-se que $57,8 \%$ dos idosos eram do sexo feminino, $54,6 \%$ tinham mais de 70 anos, 56\% eram afrodescendentes (se somados negros e pardos), 54,7\% não tinham cônjuges, 57,9\% eram analfabetos, $76,6 \%$ eram aposentados, $78,1 \%$ tinha renda familiar de 1 a 3 salários mínimos.

Os principais diagnósticos de enfermagem levantados foram: estilo de vida sedentário, presente em 53 (82,8\%) idosos; seguido de interação social prejudicada, 50 (78,1\%); e dentição prejudicada, 46 (71,8\%).

\section{DISCUSSÃO}

Destaca-se que, Na população idosa, há predominância de mulheres, e tentativas têm sido feitas para explicar essa diferença. Algumas hipóteses sugerem que os homens têm as mais altas taxas de mortalidade relacionadas à violência, acidentes de trânsito e doenças crônicas. Já as mulheres têm as mais altas taxas de morbidade em quase todas as doenças crônicas não fatais. Além disso, elas são mais inclinadas a prestar atenção aos sinais e sintomas e a procurar assistência mais frequentemente que os homens ${ }^{7}$.

Em relação à raça referida, encontrou-se $44 \%$ de brancos, $25 \%$ de negros, $31 \%$ de pardos. Considerando a soma de negros e pardos, tem-se um total de $56 \%$ de idosos afrodescendentes.

Quanto ao estado civil, 45,3\% dos idosos eram casados, $36 \%$ viúvos, $14 \%$ solteiros e 4,7\% separados. Considerando o percentual de viúvos, solteiros e separados, encontrou-se $54,7 \%$ de idosos sem companheiro. Diversos estudos têm chamado a atenção para a importância do apoio social na vida dos idosos, dentre os

\begin{tabular}{lrrr}
20 & 74,1 & 49 & 76,6 \\
05 & 18,5 & 08 & 12,5 \\
-- & ---- & 02 & 3,1 \\
-- & --- & 03 & 4,7 \\
02 & 7,4 & 02 & 3,1 \\
27 & 100,0 & 64 & 100,0 \\
22 & & & \\
05 & 81,4 & 50 & 78,1 \\
27 & 18,6 & 14 & 21,9 \\
\hline
\end{tabular}

quais destacam-se os que foram realizados com idosos atendidos pela Estratégia Saúde da Família. As redes sociais formadas por familiares e amigos significativamente reduzem os efeitos do estresse nos indivíduos mais velhos; elas oferecem suporte social na forma de amor, afeição, preocupação e assistência. Pessoas que não têm esse tipo de suporte tendem a ter mais dificuldade para lidar com o estresse. que aquelas que têm 0 suporte social. Normalmente, a A ausência de parentes, por sua vez, especificamente parentes os mais próximos, tais como o cônjuge ou os filhos, está associada com doença e mortalidade entre pessoas idosas $^{8,9}$.

Há baixo nível de escolaridade nos pesquisados, sendo que apenas $42,1 \%$ dos idosos estudados são alfabetizados, os demais, 57,9\%, são analfabetos. O nível educacional é um dos indicadores na caracterização do perfil socioeconômico da população. No caso da população idosa, o indicador de alfabetização é considerado um termômetro das políticas educacionais brasileiras do passado. Da década de 1930 até, pelo menos, os anos 1950, o ensino fundamental ainda era restrito a segmentos sociais específicos. Nessa medida, o baixo saldo da escolaridade média dessa população é um reflexo desse acesso desigual ${ }^{10}$.

Tratando-se da ocupação dos idosos, observou-se que 49 (76,6\%) eram aposentados e um baixo percentual exercia atividades laborais. Nesse sentido, estudos evidenciam que a presença do idoso aposentado favorece as relações de transferências intergeracionais, haja vista que, com a garantia de renda fixa e por meio 
da corresidência, propiciam-se as práticas de transferências de recursos em ambas as direções, dos filhos adultos para os pais idosos e vice-versa. No entanto, importa destacar que muitas dessas relações não se estabelecem de forma pacífica e harmoniosa, como o caso de idosos aposentados que são explorados e sofrem violência, ou são abandonados por parte de seus familiares, muitas vezes devido a diferenças de interesses, apresentando-se como um conflito potencial entre os membros da família11,12.

Quanto à renda familiar, observou-se que $50(78,1 \%)$ tinham renda familiar de $1 \mathrm{a}$ 3 salários mínimos. Possivelmente, a renda familiar representa um forte indicador na qualidade de vida do indivíduo, pois esta interfere diretamente na possibilidade de aquisição de bens e consumo, alimentação, moradia e outros. Tratando-se de idosos em muitas situações, a renda familiar atua como suporte em tratamentos de saúde, compra de medicamentos, entre-outros ${ }^{12}$.

Nesta etapa, se descreverá os diagnósticos de enfermagem encontrados segundo a Taxanomia II; estes foram divididos entre os 12 domínios propostos pela linguagem para facilitar sua categorização. 0 diagnóstico de enfermagem prevalente foi estilo de vida sedentário, presente em 53 (82,8\%) idosos; seguido de interação social prejudicada, 50 (78,1\%) e dentição prejudicada, 46 (71,8\%).

Inserido no domínio 1 - Controle ineficaz do regime terapêutico foi identificado em 35 (54,6\%) idosos; trata-se de um diagnóstico muito útil para a enfermagem na maioria das instituições. Os indivíduos e as famílias, apresentando uma série de problemas de saúde, agudos ou crônicos, geralmente enfrentam programas de tratamento que exigem mudanças no funcionamento ou no estilo de vida. Ainda na perspectiva da promoção da saúde, o diagnóstico manutenção ineficaz de saúde foi verificado em 25 (39,6\%) idosos. Esse diagnóstico pressupõe a incapacidade do indivíduo de identificar, controlar e/ou buscar ajuda para manter sua saúde 6 .
Fatores relacionados a esse diagnóstico: falta de apoio familiar ao idoso, prejuízo cognitivo, incapacidade de assumir responsabilidade sobre sua saúde, dentre outros. Manutenção do lar prejudicada foi observada em 21 idosos (32,8\%), sendo definida como incapacidade de manter de forma independente um ambiente seguro que promova crescimento ${ }^{6}$. Nesse sentido, o papel do enfermeiro da Estratégia Saúde da Família junto à comunidade é fundamental para apoiar os idosos, seu cuidador e família.

No domínio 2 - Nutrição, na classe um, que define os diagnósticos de ingestão, foram encontrados 37 (57,8\%) idosos com nutrição desequilibrada: menos do que as necessidades corporais; 18 (28,1\%) com nutrição desequilibrada: mais do que as necessidades corporais; e $40(62,5 \%)$ com risco para glicemia instável. Esse diagnóstico representa uma condição de risco de variação dos níveis de glicose no sangue em relação aos parâmetros normais. Vários fatores estão relacionados, tais como: aumento de peso, falta de conhecimento sobre o controle da diabetes, falta de aceitação do diagnóstico, inatividade física ${ }^{6} \mathrm{e}$ outros.

No domínio 3 - eliminação e trocas, que se refere à secreção e excreção dos produtos residuais do metabolismo do organismo, o diagnóstico constipação foi observado em 28 (43,7\%) idosos. Existem vários fatores epidemiológicos de risco para o desenvolvimento da constipação, como idade, sexo feminino, baixo nível socioeconômico, baixo consumo de fibras na dieta e estilo de vida dos países industrializados. A modificação dos hábitos alimentares gerada pela tecnologia tem introduzido o consumo de alimentos refinados desprovidos de fibras vegetais, contidas em maior quantidade nas cascas das frutas e legumes ${ }^{13}$. Nesse sentido, a avaliação nutricional e o consumo alimentar devem ser levados em consideração pelo enfermeiro na Estratégia Saúde da Família, como propõe o Caderno de Atenção Básica envelhecimento e saúde da pessoa idosa ${ }^{3}$.

Inserido no domínio 4 atividade/repouso, o diagnóstico insônia foi 
identificado em $36 \quad(56,2 \%)$ idosos. Verificou-se que 53 (82,8\%) idosos possuíam estilo de vida sedentário. 0 elevado número de idosos identificados com estilo de vida sedentário deve-se ao fato de que estes reduzem involuntariamente seu nível habitual de atividade para o que concorrem vários fatores, tais como: depressão, instabilidade musculoesquelética e outros problemas de saúde associados que limitam a mobilidade. A atividade física regular e a adoção de um determinado estilo de vida são necessários para a promoção da saúde e da qualidade de vida durante o processo de envelhecimento. Nesse sentido, a atividade física planejada e compatível com as diferenças individuais pode ter efeito redutor no ritmo dessas perdas ${ }^{14}$. Na classe equilíbrio de energia, foram identificados 19 (29,6\%) idosos com diagnóstico de fadiga, queixa extremamente comum no idoso. Caracteriza-se a fadiga, por incapacidade de mobilizar energia necessária para as atividades habituais, associada à necessidade de descansar ou dormir6.

No domínio 5 - percepção/cognição, o diagnóstico de enfermagem memória prejudicada foi identificado em $30(46,8 \%)$ idosos. Esse diagnóstico refere-se ao estado em que o indivíduo apresenta uma incapacidade temporária ou permanente de lembrar ou recordar porções de informação ou habilidades do comportamento. Alguns fatores podem estar relacionados, tais como: alterações ambientais excessivas, distúrbio neurológico, déficit auditivo, fadiga, nível educacional, entre outros ${ }^{15}$. Ainda na classe cognição, verificou-se que $25,0 \%$ dos idosos tinham comunicação verbal prejudicada, que é a habilidade diminuída, retardada ou ausente para receber, processar, transmitir e usar um sistema de símbolos ${ }^{15}$.

No domínio 6 - autopercepção verificou-se que $36(56,2 \%)$ idosos tinham risco de solidão. Esse diagnóstico refere-se ao risco do indivíduo de vivenciar desconforto associado a um desejo ou necessidade de mais contato com outros. Neste sentido, é possível mencionar fatores de risco tais como: falta de energia, isolamento físico, isolamento social, privação afetiva ${ }^{16}$.
Em relação ao domínio 7 - papéis e relacionamentos se observaram na classe desempenho de papéis, que $50 \quad(78,1 \%)$ idosos apresentavam interação social prejudicada. Essa condição pode estar relacionada à ausência de pessoas do mesmo grupo etário para interagir com os idosos e a mobilidade física limitada destes. Para idosos que convivem com situações de agravos à saúde, sair de casa pode representar um grande desafio, fazendo com que estes permaneçam quase que estritamente dentro de casa, diminuindo as oportunidades de interação social ${ }^{17}$.

0 domínio 8 - referente à sexualidade, cuja classe é a função sexual, mostrou que $32 \quad(50,0 \%)$ idosos apresentavam o diagnóstico de enfermagem padrões de sexualidade ineficazes, estado em que o indivíduo apresenta ou está em risco de apresentar mudança na saúde sexual. A saúde sexual é a integração dos aspectos somáticos, emocionais, intelectuais e sociais do ser sexual, de forma enriquecedora e fortalecedora da personalidade, da comunicação e do amor. Assim, a sexualidade é um elemento fundamental para uma boa qualidade de vida dos idosos, porém se faz necessário o conhecimento de como eles a percebem e vivenciam, permitindo a obtenção de informações relativas ao tema que possam subsidiar os profissionais de saúde com vistas ao planejamento de ações específicas objetivando a atenção integral ${ }^{18}$.

No domínio 9 - enfrentamento / tolerância ao estresse, o diagnóstico de ansiedade foi verificado em 22 (34,3\%) idosos. Esse diagnóstico está inserido na classe reações de enfrentamento, podendo ser definido como um vago sentimento de desconforto ou temor acompanhado por resposta autonômica e sentimento de apreensão causado pela antecipação do perigo ${ }^{6}$.

No domínio 10 - princípios de vida, classe, coerência entre valores/crenças/atos, o diagnóstico mais frequente foi falta de adesão, presente em $30(46,8 \%)$ idosos. A definição de falta de adesão varia conforme a 
fonte utilizada. De um modo geral, expressa o comportamento da pessoa ou cuidador que deixa de coincidir com um plano de promoção da saúde ou terapêutico acordado entre a pessoa ou cuidador e o profissional de saúde 6,16 .

No domínio 11 - segurança/ proteção, observou-se que 46 (71,8\%) idosos possuíam dentição prejudicada. Esse diagnóstico pertence à classe de lesão física. Refere-se a distúrbios nos padrões de desenvolvimento/erupção dentário ou na integridade estrutural dos dentes de um indivíduo ${ }^{16}$.

Ainda nesse domínio e classe, verificou-se a presença do diagnóstico risco de quedas em 30 (46,8\%) idosos, devendose a fatores intrínsecos como uso de medicação ansiolítica, antidepressivos, dificuldade visual, insônia, dificuldade de marcha e equilíbrio prejudicado; e a fatores extrínsecos, tais como: presença de escadas e pisos escorregadios, tapetes soltos, calçado inapropriado, ambiente com móveis e objetos em excesso, pouca iluminação, entre outros. As quedas em idosos têm como consequências, além de possíveis fraturas e risco de morte, o medo de cair novamente, a restrição de atividades, o declínio na saúde e o aumento do risco de institucionalização.

No domínio 12 - conforto, classe conforto físico, observou-se o diagnóstico de dor crônica presente em 54,6\% dos idosos, revelando que estes vivem seu dia a dia com algum grau de sofrimento, o que os impede de realizar atividades básicas e interagir com outras pessoas, podendo levar ao isolamento social.

A dor crônica como uma doença e não um sintoma pode ter consequências na qualidade de vida. Fatores como depressão, incapacidade física e funcional, dependência, afastamento social, mudanças na sexualidade, alterações na dinâmica familiar, desequilíbrio econômico, desesperança, sentimento de morte e outros encontram-se associados a quadros de dor crônica. A dor passa a ser o centro, direciona e limita as decisões e comportamentos do indivíduo. Acarreta, ainda, fadiga, anorexia, alterações do sono, constipação, náuseas, dificuldade de concentração, e outros ${ }^{19}$.

\section{CONCLUSÃO}

0 presente estudo teve como finalidade caracterizar os diagnósticos de enfermagem mais frequentes em idosos assistidos numa unidade da Estratégia Saúde da Família, tendo como referencial a Taxonomia II, da NANDA. Foi possível constatar que a maioria dos idosos que participaram do estudo era do sexo feminino, constituída por afrodescendentes, sem cônjuge, analfabetas, aposentadas, com renda familiar de 1 a 3 salários mínimos. Os diagnósticos de enfermagem prevalentes foram: estilo de vida sedentário, interação social prejudicada e dentição prejudicada; porém, em todos os 12 domínios, verificou-se alterações em termos da classificação NANDA II.

Destaca-se a importância desse mapeamento para maior precisão na escolha dos diagnósticos da Taxonomia II da NANDA e as intervenções mais adequadas nestes, a fim de se oferecer uma melhor atenção ao idoso.

Considera-se que o profissional enfermeiro, inserido na Atenção Primária à Saúde (APS), deve analisar a adequação dessa linguagem (NANDA) ao referencial de Processo de Enfermagem adotado para cada realidade. Assim, a assistência de enfermagem na Estratégia Saúde da Família por meio dessa metodologia tende a ser eficiente e efetiva, e metas podem ser alcançadas com base na sistematização do Processo de Enfermagem, que deve ser compreendido como o processo metodológico para o desempenho da prática profissional dos enfermeiros na APS.

\section{REFERÊNCIAS}

1. Ministério do Planejamento, Orçamento e Gestão (Br). Instituto Brasileiro de Geografia e Estatística. Diretoria de Pesquisas. Coordenação de População e Indicadores Sociais. Síntese dos indicadores sociais: uma analise das condições de vida da população brasileira em 2010. Rio de Janeiro: IBGE; 2010. p. 191-7.

2. Veras RP. Terceira idade: gestão contemporânea em saúde. Rio de Janeiro: Relume-Dumará; 2002. 
3. Ministério da Saúde (Br). Secretaria de Atenção à Saúde. Departamento de Atenção Básica. Envelhecimento e saúde da pessoa idosa. Brasília: Ministério da Saúde; 2006. 192 p.

4. Chiesa AM, Fracolli EA, Sousa MF. Enfermagem, academia e saúde da família: diálogo possível em torno da formação e a defesa da equidade como eixo norteador. Rev. Bras. Saúde Fam. 2002; 2(4): 52-9.

5. Smeltzer SC, Suddarth BGB. Tratado de Enfermagem Médico-Cirúrgica. Rio de Janeiro:Guanabara Koogan; 2003.

6. Carpenitto LJ. Diagnósticos de enfermagem: aplicação à prática clínica. 8 ed. Porto Alegre: Artes Médicas; 2002.

7. Fundação Sistema Estadual de Análise de Dados (SP). Esperança de vida aumenta e diferença entre gêneros diminui: queda de homicídios em jovens poupa vidas e explica avanço masculino. [Internet]. São Paulo: SEAD; 2013 [citado em 30 ago. 2013]. Disponível

em: http://www.seade.gov.br/produtos/espvida/espvi da_jan2006.pdf.

8. Pinto JLG, Garcia ACO, Bocchi SCM, Carvalhaes MABL. Características do apoio social oferecido a idosos de área rural assistida pelo PSF. Ciênc Saúde Coletiva. 2006; 11(3):753-64.

9. Guedea MTD. Relação do bem-estar subjetivo, estratégias de enfrentamento e apoio social em idosos. Psicol. Reflex. Crit. 2006; 19(2):301-8.

10. Santos GS, Cianciarullo TI. Perfil sociodemográfico de idosos de uma área de abrangência do Programa Saúde da Família do município de Guarulhos-SP. Saúde Coletiva 2009; 33(6):200-6.

11. Tavares VO, Teixeira KMD, Wajnman S, Loreto MDS. Interfaces entre a renda dos idosos aposentados rurais e o contexto familiar. Textos e Contextos. 2011; 10(1):94- 108.

12. D'orsi E, Xavier AJ, Ramos LR. Trabalho, suporte social e lazer protegem idosos da perda funcional: estudo Epidoso. Rev. Saúde Pública. 2011; 45(4):685-92.

13. André SB, Rodriguez TN, Moraes JP. Constipação intestinal. RBM Rev. Bras. Med. [Internet]. $2000 \mathrm{dez}$ [acesso em17 set 2013]; 57(N espec). Disponível em: http://www.moreirajr.com.br/revistas.asp?id_mat eria $=584 \&$ fase $=$ imprime.

14. Buriti MSL, Campelo CG. Atividade física e envelhecimento. In: Witter GP. Envelhecimento: referenciais teóricos e pesquisas. 2 ed. Campinas: Alínea; 2010.

15. Doenges ME, Moorhouse MF, Geissler-Murr AC. Nurse's pocket guide: diagnoses, interventions, and rationales. 11th ed. Philadelphia: F.A. Davis; 2008.

16. North American Nursing Diagnosis Association. Diagnósticos de enfermagem NANDA: definições e classificações, 2009-2011. Porto Alegre: Artmed; 2011.

17. Rodrigues R, Ribeiro VS, Godoy GS, Cavalcante AMRZ, Stival MM, Lima LR. Diagnósticos de enfermagem de idosos cadastrados em Estratégias de Saúde da Família em um município do interior de Goiás. R. Enferm. Cent. O. Min. 2011; 1(2):24859.

18. Moura I, Leite MT, Hildebrandt LM. Idosos e sua percepção acerca da sexualidade na velhice. Rev. Bras. Ciênc. Envelhec. Hum. 2008; 5(2):132-40.

19. Ferrel BA, Ferrel BR, Osterweil D. Pain in the nursing home. J Am. Geriatr. Soc. 2003; 38(4):40914.

\section{CONTRIBUIÇÕES}

Gerson de Souza Santos e Marina Borges

Teixeira tiveram iguais contribuições no que se refere a:

1. Concepção e delineamento do estudo;

2. Análise e interpretação dos dados;

3. Redação do manuscrito;

4. Aprovação final da versão do manuscrito a ser publicada. 